\title{
Hydrogen sulfide therapy attenuates the inflammatory response in a porcine model of myocardial ischemia/reperfusion injury
}

\author{
Neel R. Sodha, MD, ${ }^{\mathrm{a}}$ Richard T. Clements, PhD, ${ }^{\mathrm{a}}$ Jun Feng, MD, PhD, ${ }^{\mathrm{a}}$ Yuhong Liu, MD, ${ }^{\mathrm{a}}$ \\ Cesario Bianchi, MD, PhD, ${ }^{\mathrm{a}}$ Eszter M. Horvath, MD, ${ }^{\mathrm{b}}$ Csaba Szabo, MD, PhD,${ }^{\mathrm{c}}$ Gregory L. Stahl, PhD,${ }^{\mathrm{d}}$ and \\ Frank W. Sellke, MD
}

\begin{abstract}
Introduction: Hydrogen sulfide is produced endogenously in response to myocardial ischemia and thought to be cardioprotective. The mechanism underlying this protection has yet to be fully elucidated, but it may be related to sulfide's ability to limit inflammation. This study investigates the cardioprotection provided by exogenous hydrogen sulfide and its potential anti-inflammatory mechanism of action.
\end{abstract}

\begin{abstract}
Methods: The mid left anterior descending coronary artery in 14 Yorkshire swine was acutely occluded for 60 minutes, followed by reperfusion for 120 minutes. Controls $(\mathrm{n}=7)$ received placebo, and treatment animals $(\mathrm{n}=$ 7) received sulfide 10 minutes before and throughout reperfusion. Hemodynamic and functional measurements were obtained. Evans blue and triphenyl tetrazolium chloride staining identified the area at risk and infarction. Coronary microvascular reactivity was assessed. Tissue was assayed for myeloperoxidase activity and proinflammatory cytokines.
\end{abstract}

Results: Pre-ischemia/reperfusion hemodynamics were similar between groups, whereas post-ischemia/reperfusion mean arterial pressure was reduced by $28.7 \pm 5.0 \mathrm{~mm} \mathrm{Hg}$ in controls versus $6.7 \pm 6.2 \mathrm{~mm} \mathrm{Hg}$ in treatment animals $(P=.03)$. Positive first derivative of left ventricular pressure over time was reduced by $1325 \pm 455 \mathrm{~mm}$ $\mathrm{Hg} / \mathrm{s}$ in controls versys $416 \pm 207 \mathrm{~mm} \mathrm{Hg} / \mathrm{s}$ in treatment animals $(P=.002)$. Segmental shortening in the area at risk was better in treatment animals. Infarct size (percent of area at risk) in controls was $41.0 \% \pm 7.8 \%$ versus $21.2 \% \pm 2.5 \%$ in the treated group $(P=.036)$. Tissue levels of interleukin 6 , interleukin 8 , tumor necrosis factoralpha, and myeloperoxidase activity decreased in the treatment group. Treated animals demonstrated improved microvascular reactivity.

Conclusions: Therapeutic sulfide provides protection in response to ischemia/reperfusion injury, improving myocardial function, reducing infarct size, and improving coronary microvascular reactivity, potentially through its anti-inflammatory properties. Exogenous sulfide may have therapeutic utility in clinical settings in which ischemia/reperfusion injury is encountered.

Acute myocardial infarction is the leading cause of mortality in the industrialized world, accounting for over 600,000 deaths annually in the United States alone. Re-establishment of blood flow to the ischemic region is essential to salvage ischemic myocardium from inevitable necrosis, and it is generally performed by thrombolysis, percutaneous coronary intervention, or coronary artery bypass grafting. Unfortunately, the therapeutic intervention for acute myocardial ischemia results in iatrogenic injury to myocardium, de-

\footnotetext{
From the Division of Cardiothoracic Surgery, ${ }^{\mathrm{a}}$ Beth Israel Deaconess Medical Center, Harvard Medical School, Boston, Mass; the Department of Surgery, ${ }^{\mathrm{b}}$ University of Medicine and Dentistry of New Jersey, Newark, NJ; Ikaria, Inc, ${ }^{\mathrm{c}}$ Seattle, Wash; and the Center for Experimental Therapeutics \& Reperfusion Injury, ${ }^{\mathrm{d}}$ Brigham \& Women's Hospital, Harvard Medical School, Boston, Mass.

Supported by National Heart, Lung, and Blood Institute grant HL69024-02. N.R.S. and R.T.C. are supported in part by National Institutes of Health grant T-32HL076130-02 and the Irving Bard Memorial Fellowship. Partial funding for this project was provided by Ikaria, Inc, Seattle, Wash.

Received for publication March 15, 2008; revisions received July 2, 2008; accepted for publication Aug 7, 2008; available ahead of print June 15, 2009.

Address for reprints: Frank W. Sellke, MD, 110 Francis St, LMOB 2A, Boston, MA 02215 (E-mail: fsellke@caregroup.harvard.edu).

J Thorac Cardiovasc Surg 2009;138:977-84

$0022-5223 / \$ 36.00$

Copyright (c) 2009 by The American Association for Thoracic Surgery

doi:10.1016/j.jtcvs.2008.08.074
}

scribed as "reperfusion injury."1 The combination of ischemia and subsequent reperfusion injury (I/R injury) can result in additional damage to the heart independent of the ischemic insult manifesting as post-I/R dysrhythmia, ${ }^{2}$ myocardial stunning, ${ }^{3}$ and continued myocardial ischemia resulting from endothelial damage leading to coronary microvascular dysfunction. ${ }^{4}$

Research into the pathogenesis of myocardial I/R injury has identified an endogenous inflammatory response and the release of damaging free radicals and oxidants associated with this response to be key in inducing myocardial damage. ${ }^{5}$ Key to this inflammatory response are neutrophils, which have been shown to become activated in response to $\mathrm{I} / \mathrm{R}$ injury, localizing to the site of ischemia and subsequently releasing damaging free radicals and proinflammatory mediators. ${ }^{6}$

Recently, investigation into the gaseous signaling molecule hydrogen sulfide $\left(\mathrm{H}_{2} \mathrm{~S}\right)$ has demonstrated that it may in fact serve as an endogenous mediator to limit inflammation and free radical damage. ${ }^{7}$ Specifically, $\mathrm{H}_{2} \mathrm{~S}$ has been shown to limit neutrophil adhesion and activation in response to inflammatory stimuli, as well as to suppress the release of the proinflammatory mediator tumor necrosis 


$$
\begin{aligned}
& \text { Abbreviations and Acronyms } \\
& \begin{aligned}
\mathrm{H}_{2} \mathrm{~S} & =\text { hydrogen sulfide } \\
\mathrm{IL} & =\text { interleukin } \\
\mathrm{I} / \mathrm{R} & =\text { ischemia/reperfusion } \\
\mathrm{K}_{\mathrm{ATP}} & =\text { kidney adenosine triphosphate } \\
\mathrm{LAD} & =\text { left anterior descending } \\
\mathrm{LV} & =\text { left ventricular } \\
\mathrm{LV} \mathrm{dP} / \mathrm{dt} & =\text { first derivative of left ventricular } \\
& \text { pressure over time } \\
\mathrm{MPO} & =\text { myeloperoxidase } \\
\mathrm{TNF}-\alpha & =\text { tumor necrosis factor-alpha }
\end{aligned}
\end{aligned}
$$

factor-alpha (TNF- $\alpha){ }^{8,9}$ In addition to its effects on neutrophils, $\mathrm{H}_{2} \mathrm{~S}$, which is a strong reducing agent, is able to react with multiple oxidant stressors including superoxide radical anion, ${ }^{10}$ hydrogen peroxide, ${ }^{11}$ and peroxynitrite. ${ }^{7}$

$\mathrm{H}_{2} \mathrm{~S}$, which is synthesized endogenously from L-cysteine via cystathionine- $\gamma$-lyase in the heart and vasculature, has also become the subject of recent investigation in the context of myocardial protection. These studies, which have all used rodent models of myocardial injury, have demonstrated that sulfide is able to limit myocardial infarction size and oxidative stress in response to I/R injury. ${ }^{11-13}$ On the basis of these investigations, we have tested whether administration of exogenous $\mathrm{H}_{2} \mathrm{~S}$ would limit the inflammatory response to acute myocardial $\mathrm{I} / \mathrm{R}$ injury. We used a preclinical large animal model of acute myocardial ischemia followed by reperfusion to obtain both functional and molecular data in to the myocardial protection provided by $\mathrm{H}_{2} \mathrm{~S}$ therapy.

\section{MATERIAL AND METHODS \\ Animals}

Animals were housed individually and provided with laboratory chow and water ad libitum. All experiments were approved by the Beth Israel Deaconess Medical Center animal care and use committee and the Harvard Medical Area standing committee on animals (institutional animal care and use committee) and conformed to the US National Institutes of Health guidelines regulating the care and use of laboratory animals (NIH publication 5377-3, 1996).

\section{Experimental Design}

Yorkshire pigs of either sex $(35-40 \mathrm{~kg})$ were divided randomly into control $(n=7)$ and sulfide treatment $(n=7)$ groups. Animals were subjected to regional left ventricular (LV) ischemia by occlusion of the left anterior descending (LAD) artery distal to the second diagonal branch for 60 minutes. The treatment group received sodium sulfide $\left(100 \mu \mathrm{g} / \mathrm{kg}\right.$ bolus $+1 \mathrm{mg} \cdot \mathrm{kg}^{-1}$ $\cdot \mathrm{h}^{-1}$ infusion) 10 minutes before the onset of reperfusion, whereas the control group received a placebo carrier solution of equal volume. Sodium sulfide was produced and formulated to $\mathrm{pH}$ neutrality and iso-osmolarity by Ikaria Inc (Seattle, Wash) using $\mathrm{H}_{2} \mathrm{~S}$ gas (Mattheson, Newark, Calif) as the starting material. The myocardium was reperfused for 120 minutes after ischemia. Arterial blood gas, arterial blood pressure, hematocrit value, LV pressure, heart rate, electrocardiogram, oxygen saturation, core temperature, and intravenous fluid requirements were measured and recorded. Myocardial segmental shortening in the long axis (parallel to the LAD) and short axis (perpendicular to the LAD) were recorded at baseline before the onset of ischemia and before harvest after 120 minutes of reperfusion. At the completion of the protocol, the heart was excised, and tissue samples from the ischemic-reperfused distal LAD territory were collected for molecular analyses as described herein.

\section{Surgical Protocol}

Swine were sedated with ketamine hydrochloride $(20 \mathrm{mg} / \mathrm{kg}$, intramuscularly; Abbott Laboratories, North Chicago, Ill) and anesthetized with a bolus infusion of thiopental sodium (Baxter Healthcare Corporation, Inc, Deerfield, Ill; $5.0-7.0 \mathrm{mg} / \mathrm{kg}$ intravenously), followed by endotracheal intubation. Ventilation was begun with a volume-cycled ventilator (model Narkomed II-A; North American Drager, Telford, Pa; oxygen, $40 \%$; tidal volume, $600 \mathrm{~mL}$; ventilation rate, 12 breaths/min; positive end-expiratory pressure, $3 \mathrm{~cm}$ $\mathrm{H}_{2} \mathrm{O}$; inspiratory/expiratory time, $1: 2$ ). General endotracheal anesthesia was established with 3.0\% sevoflurane (Ultane; Abbott Laboratories) at the beginning of the surgical preparation and then maintained with $1.0 \%$ throughout the experiment. One liter of lactated Ringer intravenous fluid was administered after induction of anesthesia and continued thereafter throughout the surgical protocol at $150 \mathrm{~mL} / \mathrm{h}$. A right groin dissection was performed and the femoral vein and common femoral artery were isolated and cannulated with $8 \mathrm{~F}$ sheaths (Cordis Corporation, Miami, Fla). The femoral vein was cannulated for intravenous access and drug/placebo delivery, and the right common femoral artery was cannulated for arterial blood sampling and continuous intra-arterial blood pressure monitoring (Millar Instruments, Inc, Houston, Tex). A median sternotomy was performed exposing the pericardial sac, which was then opened to form a pericardial cradle. A catheter-tipped manometer (Millar Instruments, Inc) was introduced through the apex of the left ventricle to record $L V$ pressure. Segmental shortening in the area at risk was assessed with a sonometric digital ultrasonic crystal measurement system (Sonometrics Corp, London, Ontario, Canada) using four 2-mm digital ultrasonic probes implanted in the subepicardial layer approximately $10 \mathrm{~mm}$ apart within the ischemic LV area. Cardiosoft software (Sonometrics Corp) was used for data recording (first derivative of LV pressure over time [LV dP/ $\mathrm{dt}$ ], segmental shortening, arterial blood pressure, heart rate) and subsequent data analysis to determine myocardial function. Baseline hemodynamic, functional measurement (global, $+\mathrm{LV} \mathrm{dP/dt;} \mathrm{regional,} \mathrm{segemental} \mathrm{shortening),} \mathrm{ar-}$ terial blood gas analysis, and hematocrit value were obtained. Arterial blood gas analysis was continued every 15 minutes throughout the protocol and hematocrit value was measured every 20 minutes. All animals received $75 \mathrm{mg}$ of lidocaine and $20 \mathrm{mEq}$ of potassium chloride as prophylaxis against ventricular dysrhythmia, as well as $60 \mathrm{U} / \mathrm{kg}$ of intravenous heparin bolus before occlusion of the LAD. The LAD coronary artery was occluded $3 \mathrm{~mm}$ distal to the origin of the second diagonal branch with a Rommel tourniquet. Myocardial ischemia was confirmed visually by regional cyanosis of the myocardial surface. Fifty minutes after the initiation of regional ischemia (10 minutes before the onset of reperfusion), control pigs received a placebo carrier solution infusion intravenously, and treatment animals received exogenous sulfide, generated as sodium sulfide (100 $\mu \mathrm{g} / \mathrm{kg}$ bolus $+1 \mathrm{mg} \cdot \mathrm{kg}^{-1} \cdot \mathrm{h}^{-1}$ infusion $)$ until the end of the experimental protocol. The Rommel tourniquet was released 60 minutes after the onset of acute ischemia and the myocardium was reperfused for 120 minutes. At the end of the reperfusion period, hemodynamic and functional measurements were recorded as described earlier. This was followed by religation of the LAD and injection of monastryl blue pigment (Engelhard Corp, Louisville, Ky) at a 1:150 dilution in phosphate-buffered saline solution into the aortic root after placement of an aortic crossclamp distal to the coronary arterial ostia to demarcate the area at risk. The heart was rapidly excised and the entire left ventricle, including the septum, was dissected free. The left ventricle was cut into $1-\mathrm{cm}$ thick slices perpendicular to the axis of the LAD. The area at risk was clearly identified by lack of blue pigment staining. Tissue from the area at risk of the slice $1 \mathrm{~cm}$ proximal to the $\mathrm{LV}$ apex was isolated and divided for use in molecular and microvascular studies. The remaining slices were weighed for infarct size calculation as described below. Ventricular dysrhythmia (ventricular fibrillation or pulseless ventricular tachycardia) events 
were recorded and treated with immediate electrical cardioversion ( $50 \mathrm{~J}$, internal paddles).

\section{Measurement of Global and Regional Myocardial Function}

Global myocardial function was assessed by calculating the maximum positive first derivative of $\mathrm{LV}$ pressure over time $(+\mathrm{dP} / \mathrm{dt})$. Regional myocardial function was determined by using subepicardial 2-mm ultrasonic probes to calculate the percentage segmental shortening, which was normalized to the baseline. Measurements were taken at baseline before the onset of ischemia and at the end of reperfusion. The ventilator was stopped during data acquisition to eliminate the effects of respiration. Measurements were made during at least three cardiac cycles in normal sinus rhythm and then averaged. Digital data were inspected for the correct identification of end-diastole and end-systole. End-diastolic segment length was measured at the onset of the $+\mathrm{dP} / \mathrm{dt}$ and the end-systolic segment length at the peak $-\mathrm{dP} / \mathrm{dt}$.

\section{Coronary Microvessel Studies}

Coronary microvessel studies were performed to examine the effects of sulfide on endothelial and vascular smooth muscle injury after I/R in the coronary microcirculation. After cardiac harvest, myocardial specimens from the ischemic LAD territory were immersed in $4^{\circ} \mathrm{C}$ Krebs solution, and coronary arterioles (80-130 $\mu \mathrm{m}$ in diameter and 1-2 $\mathrm{mm}$ in length) were dissected sharply from the surrounding tissue with a $40 \times$ magnification dissecting microscope. Microvessels were mounted and examined in a pressurized isolated organ chamber, as described previously. ${ }^{14}$ The responses to sodium nitroprusside ( $1 \mathrm{nmol} / \mathrm{L}-100 \mu \mathrm{mol} / \mathrm{L})$, an endothelium-independent cyclic guanosine monophosphate-mediated vasodilator, as well as adenosine $5^{\prime}$-diphosphate $(1 \mathrm{nmol} / \mathrm{L}-10 \mu \mathrm{mol} / \mathrm{L})$, an endothelium-dependent receptor-mediated vasodilator that acts via bioavailable nitric oxide, were studied after precontraction to $20 \%$ to $50 \%$ of the baseline diameter with the thromboxane A2 analog U46619 (0.1-1 $\mu \mathrm{mol} / \mathrm{L})$. Relaxation responses were defined as the percent relaxation of the precontracted diameter.

\section{Quantification of Myocardial Infarct Size}

The left ventricle was isolated (including septum), cut into 1-cm slices, and immediately immersed in 1\% triphenyl tetrazolium chloride (Sigma Chemical Co, St Louis, Mo) in phosphate buffer ( $\mathrm{pH} 7.4)$ at $38^{\circ} \mathrm{C}$ for 30 minutes. The infarct area (characterized by absence of staining), the noninfarcted area at risk (characterized by red tissue staining), and the nonischemic portion of the left ventricle (characterized by purple tissue staining) were sharply dissected from one another and weighed. The percentage area at risk was defined as follows:

(Infarct mass + Noninfarct area-at-risk mass) $/$ Total LV mass $\times 100$

Infarct size was calculated as a percentage of area at risk to normalize for any variation in area-at-risk size using the following equation:

$($ Infarct mass + Total mass area at risk $) \times 100$

\section{Tissue Myeloperoxidase Activity Assay}

Myocardial tissue from the distal LAD territory was harvested, and myeloperoxidase (MPO) activity was measured as previously described. ${ }^{15}$ Assessment of this assay in our laboratory demonstrated a linear relationship $(r=0.92)$ such that $1 \mathrm{U}$ of MPO activity correlated with $2.9 \times 10^{6}$ porcine neutrophils.

\section{Tissue Inflammatory Marker Quantification}

Myocardial tissue $(50 \mathrm{mg})$ from the area at risk was homogenized in RIPA buffer (Boston BioProducts, Worcester, Mass) with protease inhibitor added (Complete Tablets; Roche Applied Sciences, Indianapolis, Ind) and centrifuged at $12,000 \mathrm{~g}$ for 10 minutes. Aliquots of supernatants were obtained and a cytokine array was used (Allied Biotech Inc, Ijamsville, Md) for detection of interleukin (IL) 6, IL-8, and TNF- $\alpha$ in triplicate as described previously. ${ }^{16}$ Tissue levels of inflammatory mediators were calculated on the basis of standards provided by the manufacturer.

\section{Immunohistochemistry}

Myocardial tissue from the ischemic territory was placed in $10 \%$ buffered formalin for 24 hours, followed by paraffin mounting and sectioning into $4-\mu \mathrm{m}$ slices. For the immunohistochemical detection of nitrosative stress, rabbit polyclonal anti-nitrotyrosine antibody (Upstate Biotechnology, Lake Placid, NY) $\left(1: 80,4^{\circ} \mathrm{C}\right.$, overnight $)$ was used. Secondary labeling was achieved by using biotinylated horse anti-mouse antibody (Vector Laboratories, Burlingame, Calif) (30-min room temperature). Horseradish peroxidase-conjugated avidin (30-min, room temperature) and brown-colored diaminobenzidine (6-min, room temperature) was used to visualize the labeling (Vector Laboratories). The sections were counterstained with hematoxylin (blue color). The intensity of specific staining of individual sections was determined by a blinded experimenter. For anti-nitrotyrosine labeling, a score range between 1 and 10 was used, where $1=$ no staining and $10=$ maximal staining.

\section{Data Analysis}

Data are reported as means \pm standard error of the mean. Microvessel responses are expressed as percent relaxation of the preconstricted diameter and were analyzed by 2-way repeated-measures analysis of variance examining the relationship between vessel relaxation, log concentration of the vasoactive agent of interest, and the experimental group (SAS version 9.1, Cary, NC). Bonferroni corrections were applied to multiple tests.

\section{RESULTS \\ Survival}

Twelve of 14 animals survived until completion of the experimental protocol, with 1 animal in each group lost to irreversible dysrhythmia. Both animals were lost during the ischemic period before administration of placebo or sulfide and were excluded from further data analysis. (Functional data from 4 of 6 animals in each group have been used in a previous study published by our group using the same experimental protocol. ${ }^{17}$ )

\section{Arterial Blood Gas, Hematocrit, and Core Temperature}

No significant differences were observed between arterial $\mathrm{pH}, \mathrm{PCO}_{2}, \mathrm{Po}_{2}$, hematocrit value, or core temperature at baseline or at the end of reperfusion.

\section{Hemodynamic Parameters}

Heart rate and mean arterial blood pressure were similar between groups at baseline (heart rate: placebo $74.0 \pm 2.9$ vs sulfide $69.7 \pm 8.5$ beats $/ \mathrm{min} ; P=.64$; mean arterial pressure: placebo $63.2 \pm 2.9$ vs sulfide $56.3 \pm 3.9 \mathrm{~mm}$ $\mathrm{Hg} ; P=.19)$. Whereas heart rate remained similar between groups at the end of reperfusion (placebo $81.0 \pm 3.7 \mathrm{vs}$ sulfide $78.0 \pm 4.1$ beats $/ \mathrm{min} ; P=.60$ ), mean arterial pressure 
was significantly lower in the placebo group (placebo $34.5 \pm$ 4.1 vs sulfide $49.6 \pm 4.8 \mathrm{~mm} \mathrm{Hg} ; P=.03 *)$.

\section{Global and Regional Myocardial Function}

Global LV function as determined from $\mathrm{LV}+\mathrm{dP} / \mathrm{dt}$ was similar between groups before the onset of ischemia (placebo $2585 \pm 429 \mathrm{~mm} \mathrm{Hg} / \mathrm{s}$ vs sulfide $2517 \pm 166 \mathrm{~mm}$ $\mathrm{Hg} / \mathrm{s} ; P=.89)$. At the end of the reperfusion period, LV $\mathrm{dP} / \mathrm{dt}$ was significantly lowered in the placebo group (relative to pre-I/R LV dP/dt) compared with the sulfide-treated group (placebo $1260 \pm 152 \mathrm{~mm} \mathrm{Hg} / \mathrm{s}$ vs sulfide $2102 \pm$ $123 \mathrm{~mm} \mathrm{Hg} / \mathrm{s}$ ). This reflected a $51.5 \%$ reduction in $\mathrm{LV}$ $\mathrm{dP} / \mathrm{dt}$ in the placebo group and a $16.5 \%$ reduction in $\mathrm{LV}$ $\mathrm{dP} / \mathrm{dt}$ in the sulfide group after $\mathrm{I} / \mathrm{R}$ injury $\left(P=.002^{*}\right)$. Regional myocardial function in the area at risk was similar between groups in both longitudinal axes (placebo $13.57 \% \pm 3.04 \%$ vs sulfide $15.22 \% \pm 0.86 \%$ segmental shortening; $P=.61$ ) and horizontal axes (placebo $14.93 \%$ $\pm 1.78 \%$ vs sulfide $16.53 \% \pm 2.00 \%$ segmental shortening; $P=.56$ ) before the onset of ischemia. At the end of reperfusion, segmental shortening in the longitudinal axis was impaired significantly in both groups, but was significantly better in the sulfide-treated animals (placebo $4.98 \%$ $\pm 0.73 \%$ vs sulfide $8.95 \% \pm 1.01 \%$ segmental shortening; $P=.01^{*}$ ) (Figure 1, $A$ ). Horizontal segmental shortening was also significantly impaired in both groups at the end of reperfusion, and no significant differences were seen between groups after I/R (placebo $7.00 \% \pm 1.01 \%$ vs sulfide $7.87 \% \pm 1.66 \%$ segmental shortening; $P=.66)$ (Figure $1, B)$.

\section{Coronary Microvessel Function}

Coronary microvascular relaxation studies were performed to assess endothelial dysfunction and microvascular smooth muscle dysfunction in response to $\mathrm{I} / \mathrm{R}$ injury using adenosine 5'-diphosphate (an endothelium-dependent agent) and sodium nitroprusside (an endothelium-independent agent), respectively. Both endothelium-dependent and endothelium-independent relaxation were significantly improved in the sulfide group when compared with the placebo group (Figure 2, $A$ and $B$ ).

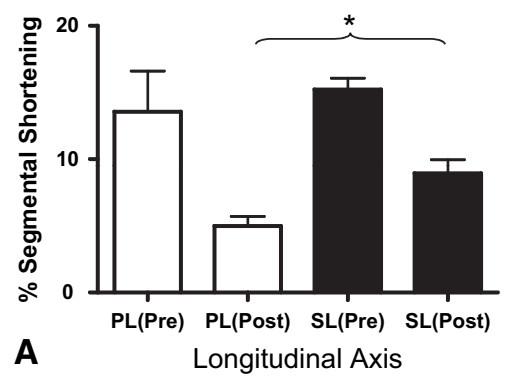

\section{Incidence of Ventricular Fibrillation/Ventricular Tachycardia}

The incidence of ventricular fibrillation or pulseless ventricular tachycardia was similar between groups during the period of ischemia (placebo $0.17 \pm 0.17$ vs sulfide $0.50 \pm$ 0.22 episodes per animal; $\mathrm{n}=0.26$ ) and during the period of reperfusion (placebo $1.50 \pm 0.50$ vs sulfide $0.83 \pm 0.31$ episodes per animal; $\mathrm{n}=0.28$ ). One animal in each group was lost to irreversible dysrhythmia during the period of ischemia and both were excluded from data analysis, as noted earlier; otherwise, all dysrhythmias were successfully terminated with electrical cardioversion.

\section{Myocardial Infarct Size}

The ischemic area at risk, as a percentage of total LV mass, was similar between groups (placebo 30.9\% \pm $2.5 \%$ vs sulfide $32.5 \% \pm 2.5 \%$ of $L V$ mass; $P=.68$ ). Myocardial infarct size was significantly reduced by 1.94 -fold in the sulfide-treated group relative to the placebo group (placebo $40.95 \% \pm 7.8 \%$ vs sulfide $21.16 \% \pm 2.5 \%$ of area at risk; $\left.P=.036^{*}\right)$ (Figure 3).

\section{Immunohistochemistry Staining for Free Radical Stress}

Nitrotyrosine staining scores were significantly lower in sulfide-treated animals (placebo $8.0 \pm 0.63$ vs sulfide 5.3 $\left.\pm 0.84 ; P=.037^{*}\right)$ (Figure 4).

\section{Tissue MPO Activity}

Tissue MPO activity, a reflection of neutrophil inflammatory activity, was markedly reduced in the sulfide-treated animals. Animals in the placebo group demonstrated $0.0397 \pm$ 0.016 units of MPO activity per milligram protein vs 0.0025 \pm 0.003 units of MPO activity per milligram protein in the sulfide-treated animals $(P=.04 *)$.

\section{Inflammatory Cytokines}

Myocardial IL-6 levels were lower in sulfide-treated animals (placebo $0.76 \pm 0.16$ vs sulfide $0.23 \pm 0.14 \mathrm{pg} / \mathrm{mL}$; $P=.03^{*}$ ), as were levels of IL-8 (placebo $0.58 \pm 0.15$ vs sulfide $0.04 \pm 0.04 \mathrm{pg} / \mathrm{mL} ; P=.003 *)$. Myocardial levels of TNF- $\alpha$ were reduced 1.33-fold in sulfide-treated animals

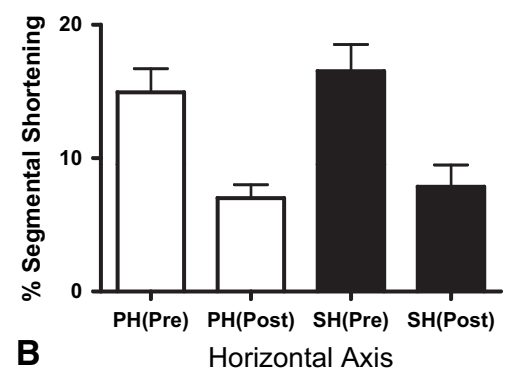

FIGURE 1. Regional LV function ( $\%$ segmental shortening) was preserved to a greater extent in sulfide-treated animals in the longitudinal axis (A), whereas no differences were observed in the horizontal axis (B). $P$, Placebo; $S$, sulfide; Pre, pre-I/R; Post, post-I/R; $L$, longitudinal axis; $H$, horizontal axis. ${ }^{*} P<.05$. 

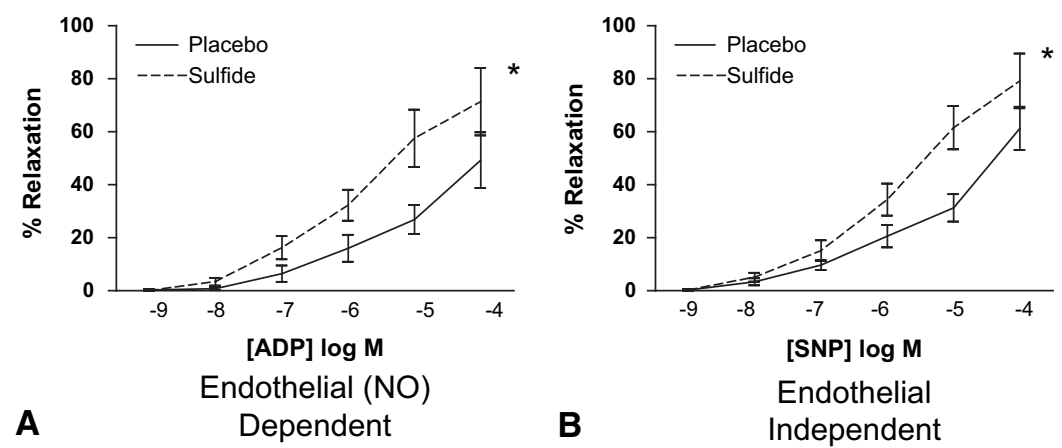

FIGURE 2. Endothelium-dependent (A) and endothelium-independent (B) coronary microvascular relaxation was improved in sulfide-treated animals. $N O$, Nitric oxide; $A D P$, adenosine $5^{\prime}$-diphosphate; $S N P$, sodium nitroprusside. $* P<.05$.

(placebo $0.52 \pm 0.11$ vs sulfide $0.16 \pm 0.09 \mathrm{pg} / \mathrm{mL} ; P=$ $.03 *)$.

\section{DISCUSSION}

The current study demonstrates that parenteral therapeutic administration of sodium sulfide before and during the reperfusion period provides significant myocardial protection in response to acute $\mathrm{I} / \mathrm{R}$ injury, limiting infarct size, improving global and regional LV function, improving coronary microvascular function, and limiting the inflammatory response to $\mathrm{I} / \mathrm{R}$ injury in a preclinical porcine model of myocardial infarction. Previous work in murine models has demonstrated that endogenous sulfide acts as a myocardial protective agent in the setting of acute ischemia. ${ }^{13}$ Johansen Ytrehus, and Baxter $^{18}$ have demonstrated the efficacy of sulfide, as an exogenously administered therapeutic agent, in an isolated rat heart model to reduce infarct size after I/R injury, whereas Zhu and associates ${ }^{12}$ have demonstrated such findings in a murine model using exteriorized hearts. Notably, the previously mentioned studies ${ }^{12,18}$ administered sulfide before the onset of ischemia. Recently, Elrod and colleagues ${ }^{19}$ have demonstrated that the therapeutic administration of sulfide at the time of reperfusion limits infarct size and inflammation and preserves myocardial function in mice subjected to I/R injury. This study, which used a murine model as well, demonstrated that sulfide therapy resulted in decreased levels of IL- $1 \beta$ but found no differences in TNF$\alpha$ or IL-10 levels. Our study, which uses a preclinical large

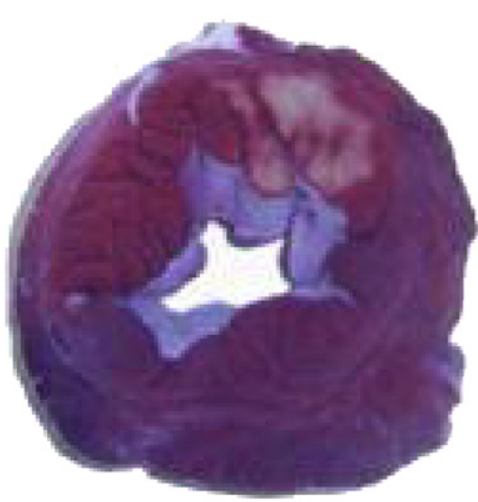

Placebo

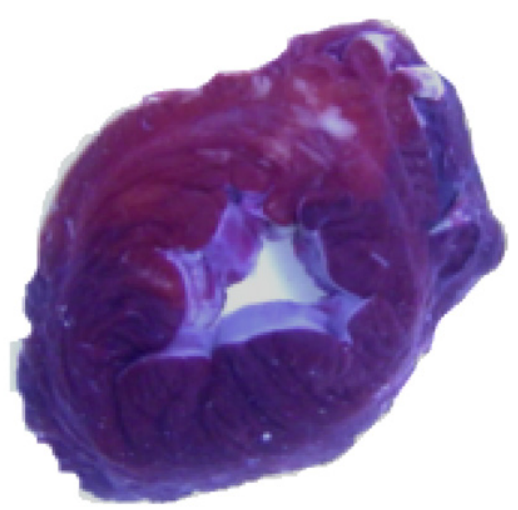

Sulfide
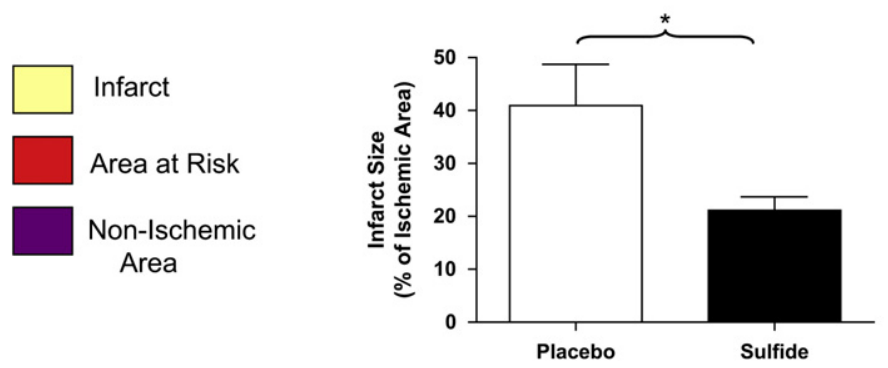

FIGURE 3. Sulfide-treated animals had significantly smaller infarct sizes than did the placebo group. $* P<.05$. 


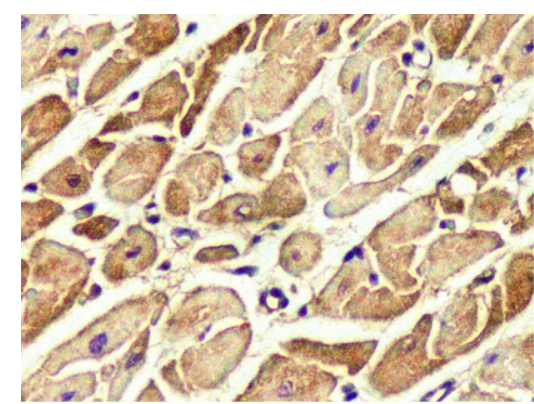

Placebo

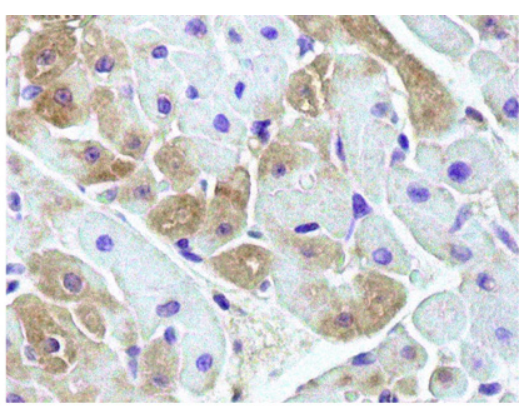

Sulfide

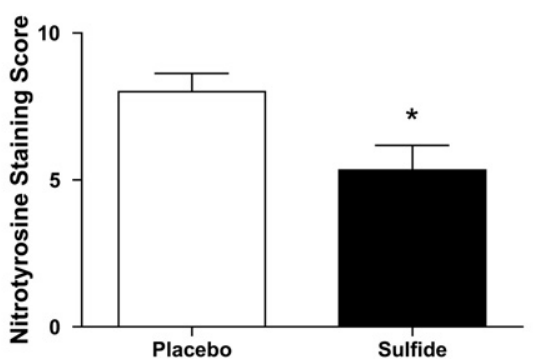

FIGURE 4. Nitrotyrosine staining intensity was significantly lower in sulfide-treated animals. ${ }^{*} P<.05$.

animal model and administers sulfide at a clinically applicable time point, is consistent with the prior studies, but notably found a significant reduction in TNF- $\alpha$, as well as IL- 6 and IL-8 levels.

$\mathrm{H}_{2} \mathrm{~S}$ is an odorless, water-soluble gas that is produced endogenously in the cardiovascular system. Although its mechanism of action has yet to be fully elucidated, three key findings thus far may relate to its ability to protect the myocardium - activation of kidney adenosine triphosphate $\left(\mathrm{K}_{\mathrm{ATP}}\right)$ channels, ${ }^{12}$ limitation of neutrophil endothelial adherence/infiltration, ${ }^{20}$ and its ability to scavenge oxidants and free radicals. ${ }^{7}$ Activation of $\mathrm{K}_{\mathrm{ATP}}$ channels has been shown to be cardioprotective. ${ }^{21}$ In vivo ${ }^{13}$ studies have shown that the myocardial protection provided by sulfide in response to myocardial ischemia is limited when $\mathrm{K}_{\mathrm{ATP}}$ channels are pharmacologically inhibited, although inhibition of $\mathrm{K}_{\mathrm{ATP}}$ channels can increase the severity of myocardial injury in control animals as well (independently from the presence or absence of exogenously administered sulfide). ${ }^{22}$ Although there is no evidence to date indicating sulfide directly augments myocardial contractility, its benefits on global and regional LV function may relate to its ability to limit myocardial injury from proinflammatory cytokines such as IL-6, IL-8, TNF- $\alpha$, and free radicals. The anti-inflammatory effects of sulfide have been demonstrated in multiple studies (noncardiac). These studies have shown that sulfide can interfere with neutrophil activity, ${ }^{7}$ suppress granulocyte adherence to the endothelium and infiltration, and suppress the expression of TNF- $\alpha .{ }^{8,20} \mathrm{IL}-6$, which is released in response to myocardial I/R injury, ${ }^{23}$ has been shown to depress myo- cardial function. ${ }^{24}$ IL-8, also released in response to myocardial I/R injury, increases neutrophil adhesion and the inflammatory response. ${ }^{25}$ TNF- $\alpha$ may play multiple roles in the pathogenesis of myocardial I/R injury, inducing endothelial cell adhesion molecules allowing for neutrophil infiltration, ${ }^{26}$ amplifying the inflammatory response, and increasing the production of reactive oxygen species, ${ }^{27}$ as well as having direct myocardial depressant and apoptotic actions. ${ }^{28}$ Tissue MPO activity, a marker of neutrophil infiltration and activity, was also significantly reduced in the myocardium of sulfide-treated animals. This is likely the result of reduced neutrophil infiltration, as has been shown with sulfide administration, ${ }^{20}$ and may protect the myocardium from damaging proteases and free radicals generated by neutrophils.

The coronary microvascular circulation, consisting of arterioles less than $175 \mu \mathrm{m}$ in diameter, is the principal site of resistance in the coronary circulation and responds to metabolic stimuli to govern myocardial perfusion. Dysfunction in this vascular bed, which is known to occur after myocardial $\mathrm{I} / \mathrm{R}$ injury, ${ }^{29}$ is thought to be responsible for the impairments in myocardial perfusion observed after re-establishment of flow in the target vessels of thrombolysis, percutaneous coronary intervention, and coronary artery bypass grafting - the large epicardial coronary arteries. ${ }^{30}$ This microcirculatory dysfunction is thought to manifest from diminished bioavailability of nitric oxide, which can be scavenged by free radicals released in the setting of I/R injury, ${ }^{31}$ as well from increased levels of proinflammatory mediators such as TNF- $\alpha$, which can impair endothelium-dependent microvascular vasorelaxation and increase circulating levels of 
free radicals. ${ }^{32}$ The improvement in endothelium-dependent vasorelaxation in our study with sulfide therapy is consistent with the reduction in TNF- $\alpha$ observed, as well as with the ability of $\mathrm{H}_{2} \mathrm{~S}$ to act as an antioxidant. Interestingly, we also observed improvements in endothelium-independent vasorelaxation in the coronary microvessels of sulfidetreated animals. This vasorelaxation in response to sodium nitroprusside, an agent active on the vascular smooth muscle, is consistent with the ability of sulfide to activate vascular smooth muscle $\mathrm{K}_{\mathrm{ATP}}$ channels, allowing for enhanced vasorelaxation. The improvements in coronary microvascular relaxation may have significant clinical relevance, as it is suggested up to $40 \%$ of patients may fail to regain appropriate myocardial perfusion despite achieving Thrombolysis in Myocardial Infarction grade 3 epicardial flow after intervention, attributed partly to coronary microvascular dysfunction. ${ }^{30,31,33}$

\section{Limitations}

Despite providing functional and molecular data into the effects of sulfide therapy in myocardial $\mathrm{I} / \mathrm{R}$ injury, this study has several limitations. Our time course for tissue harvest ( 3 hours after the onset of ischemia) is not able to account for long-term effects of sulfide on myocardial function and infarct extension. Notably, recent research has demonstrated in a murine model that sulfide therapy provides significant preservation of myocardial function in response to acute $\mathrm{I} / \mathrm{R}$ injury at 72 hours after the initial ischemic insult. ${ }^{19}$ The focus on functional data and inflammatory markers did not include a mechanistic investigation into sulfide's method of action in providing myocardial protection. Such studies would be more suitable in rodents, in which multiple dosing groups can be established and genetically modified animals are also available. The porcine model used in this study is somewhat electrically unstable given the lack of pre-formed collaterals in the porcine myocardium. This allows for creation of an acute area of ischemia with limited perfusion from collateral vessels from adjoining myocardium. Although beneficial in allowing for clear delineation of an ischemic zone, the model is subject to significant ventricular dysrhythmias from the severity of the $\mathrm{I} / \mathrm{R}$ injury. The incidence of ventricular fibrillation/ventricular tachycardia observed may lead to myocardial preconditioning, which can subsequently affect infarct size and is an inherent limitation to the porcine model used.

\section{CONCLUSIONS}

Therapeutic administration of $\mathrm{H}_{2} \mathrm{~S}$, generated as sodium sulfide, before the onset of reperfusion, markedly attenuates myocardial $\mathrm{I} / \mathrm{R}$ injury, limiting inflammation and preserving myocardial function. $\mathrm{H}_{2} \mathrm{~S}$ may have a valuable therapeutic role in the clinical setting when administered concomitantly with the coronary revascularization process.
We thank Dr James D. McCully for his valuable assistance with the surgical protocol and the staff of the Animal Research Facility at the Beth Israel Deaconess Medical Center for their efforts. The sodium sulfide solution used in the current studies was produced and formulated by Paul Hill (Ikaria Inc, Seattle, Wash).

\section{References}

1. Moens AL, Claeys MJ, Timmermans JP, Vrints CJ. Myocardial ischemia/reperfusion-injury, a clinical view on a complex pathophysiological process. Int J Cardiol. 2005;100:179-90.

2. Manning AS, Hearse DJ. Reperfusion-induced arrhythmias: mechanisms and prevention. J Mol Cell Cardiol. 1984;16:497-518.

3. Ambrosio G, Tritto I. Clinical manifestations of myocardial stunning. Coron Artery Dis. 2001;12:357-61.

4. Lefer AM, Lefer DJ. The role of nitric oxide and cell adhesion molecules on the microcirculation in ischaemia-reperfusion. Cardiovasc Res. 1996;32:743-51.

5. Roberts MJ, Young IS, Trouton TG, Trimble ER, Khan MM, Webb SW, et al. Transient release of lipid peroxides after coronary artery balloon angioplasty Lancet. 1990;336:143-5.

6. Kloner RA, Giacomelli F, Alker KJ, Hale SL, Matthews R, Bellows S. Influx of neutrophils into the walls of large epicardial coronary arteries in response to ischemia/reperfusion. Circulation. 1991;84:1758-72.

7. Whiteman M, Armstrong JS, Chu SH, Jia-Ling S, Wong BS, Cheung NS, et al. The novel neuromodulator hydrogen sulfide: an endogenous peroxynitrite 'scavenger'? J Neurochem. 2004;90:765-8.

8. Fiorucci S, Antonelli E, Distrutti E, Rizzo G, Mencarelli A, Orlandi S, et al. Inhibition of hydrogen sulfide generation contributes to gastric injury caused by anti-inflammatory nonsteroidal drugs. Gastroenterology. 2005;129:1210-24.

9. Hu LF, Wong PT, Moore PK, Bian JS. Hydrogen sulfide attenuates lipopolysaccharide-induced inflammation by inhibition of p38 mitogen-activated protein kinase in microglia. J Neurochem. 2007;100:1121-8.

10. Mitsuhashi H, Yamashita S, Ikeuchi H, Kuroiwa T, Kaneko Y, Hiromura K, et al. Oxidative stress-dependent conversion of hydrogen sulfide to sulfite by activated neutrophils. Shock. 2005;24:529-34.

11. Geng B, Chang L, Pan C, Qi Y, Zhao J, Pang Y, et al. Endogenous hydrogen sulfide regulation of myocardial injury induced by isoproterenol. Biochem Biophys Res Commun. 2004;318:756-63.

12. Zhu YZ, Wang ZJ, Ho P, Loke YY, Zhu YC, Huang SH, et al. Hydrogen sulfide and its possible roles in myocardial ischemia in experimental rats. $J$ Appl Physiol. 2007; 102:261-8.

13. Sivarajah A, McDonald MC, Thiemermann C. The production of hydrogen sulfide limits myocardial ischemia and reperfusion injury and contributes to the cardioprotective effects of preconditioning with endotoxin, but not ischemia in the rat. Shock. 2006;26:154-61.

14. Tofukuji M, Metais C, Li J, Hariawala MD, Franklin A, Vassileva C, et al. Effects of ischemic preconditioning on myocardial perfusion, function, and microvascular regulation. Circulation. 1998;98:II197-204; discussion II204-5.

15. Amsterdam EA, Stahl GL, Pan HL, Rendig SV, Fletcher MP, Longhurst JC. Limitation of reperfusion injury by a monoclonal antibody to C5a during myocardial infarction in pigs. Am J Physiol. 1995;268:H448-57.

16. Gavin MA, Rasmussen JP, Fontenot JD, Vasta V, Manganiello VC, Beavo JA, et al. Foxp3-dependent programme of regulatory T-cell differentiation. Nature. 2007;445:771-5

17. Sodha NR, Clements RT, Feng J, Liu Y, Bianchi C, Horvath EM, et al. The effects of therapeutic sulfide on myocardial apoptosis in response to ischemia-reperfusion injury. Eur J Cardiothorac Surg. 2008;33:906-13.

18. Johansen D, Ytrehus K, Baxter GF. Exogenous hydrogen sulfide (H2S) protects against regional myocardial ischemia-reperfusion injury-Evidence for a role of K ATP channels. Basic Res Cardiol. 2006;101:53-60.

19. Elrod JW, Calvert JW, Morrison J, Doeller JE, Kraus DW, Tao L, et al. Hydrogen sulfide attenuates myocardial ischemia-reperfusion injury by preservation of mitochondrial function. Proc Natl Acad Sci U S A. 2007;104:15560-5.

20. Zanardo RC, Brancaleone V, Distrutti E, Fiorucci S, Cirino G, Wallace JL. Hydrogen sulfide is an endogenous modulator of leukocyte-mediated inflammation. Faseb J. 2006;20:2118-20.

21. Lawton JS, Sepic JD, Allen CT, Hsia PW, Damiano RJ Jr. Myocardial protection with potassium-channel openers is as effective as St. Thomas' solution in the rabbit heart. Ann Thorac Surg. 1996;62:31-8; discussion 38-9.

22. Miura T, Miki T. ATP-sensitive $\mathrm{K}+$ channel openers: old drugs with new clinical benefits for the heart. Curr Vasc Pharmacol. 2003;1:251-8. 
23. Kukielka GL, Smith CW, Manning AM, Youker KA, Michael LH, Entman ML. Induction of interleukin-6 synthesis in the myocardium. Potential role in postreperfusion inflammatory injury. Circulation. 1995;92:1866-75.

24. Hennein HA, Ebba H, Rodriguez JL, Merrick SH, Keith FM, Bronstein MH, et al. Relationship of the proinflammatory cytokines to myocardial ischemia and dysfunction after uncomplicated coronary revascularization. $J$ Thorac Cardiovasc Surg. 1994;108:626-35.

25. Kukielka GL, Smith CW, LaRosa GJ, Manning AM, Mendoza LH, Daly TJ, et al. Interleukin-8 gene induction in the myocardium after ischemia and reperfusion in vivo. J Clin Invest. 1995;95:89-103.

26. Dinarello CA. Proinflammatory cytokines. Chest. 2000;118:503-8.

27. Nian M, Lee P, Khaper N, Liu P. Inflammatory cytokines and postmyocardial infarction remodeling. Circ Res. 2004;94:1543-53.

28. Herrera-Garza EH, Stetson SJ, Cubillos-Garzon A, Vooletich MT, Farmer JA, Torre-Amione G. Tumor necrosis factor-alpha: a mediator of disease progression in the failing human heart. Chest. 1999;115:1170-4.
29. Hein TW, Zhang C, Wang W, Chang CI, Thengchaisri N, Kuo L. Ischemia-reperfusion selectively impairs nitric oxide-mediated dilation in coronary arterioles: counteracting role of arginase. Faseb J. 2003;17:2328-30.

30. Ito H, Tomooka T, Sakai N, Yu H, Higashino Y, Fujii K, et al. Lack of myocardial perfusion immediately after successful thrombolysis. A predictor of poor recovery of left ventricular function in anterior myocardial infarction. Circulation. 1992;85: 1699-705.

31. Prasad A, Gersh BJ. Management of microvascular dysfunction and reperfusion injury. Heart. 2005;91:1530-2.

32. Zhang C, Xu X, Potter BJ, Wang W, Kuo L, Michael L, et al. TNF-alpha contributes to endothelial dysfunction in ischemia/reperfusion injury. Arterioscler Thromb Vasc Biol. 2006;26:475-80.

33. Prasad A, Stone GW, Aymong E, Zimetbaum PJ, McLaughlin M, Mehran R, et al. Impact of ST-segment resolution after primary angioplasty on outcomes after myocardial infarction in elderly patients: an analysis from the CADILLAC trial. Am Heart J. 2004; 147:669-75. 